you fear criticism and are reluctant to get others to review your work. Finally, there are wise words about 'early writing', and writing as you go through a project.

Section three is called 'Content and Structure', and concerns the mechanics of writing. Crucial is to 'find your story' (What's it all about?). As in a novel Heard suggests you set up an interesting and dramatic situation (by the Title and in the Introduction), tell the story (state your results), and then ensure that there is a resolution to the problem (the happy ending) in the Discussion. Heard talks of ways to ensure your story is clear and understandable, by use of wordstacks, concept maps and outlines. There follows sections on all parts of a paper (I particularly recommend his thoughts on Introduction). The advice is wonderful on the use of numbers (volume of numerical data), as is that on graphs, tables and figures-and how graphics should relate to text. Finally there is guidance on writing a Discussion, which Heard characterises and champions as 'turning data into knowledge'. Section three really should be compulsory reading for all scientific writers!

Section four details the nuts and bolts of writing scientifically, for example, the paragraph, which is 'a unit of logical organisation which is unified, coherent and distinct'. Thence follows chapters on essential matters, such as sentences, the passive or active voice, words themselves, and the wonderful Chapter 20 on Brevity, of which the first paragraph is 'Be brief'.

Revision of the manuscript is the subject of section five. There is much sage advice about the process of revision and reviews of a paper. This is divided into chapters on self-revision (a very necessary skill), friendly revision ('too often overlooked'), formal external review, and dealing with reviewers comments and editorial staff. Heard suggests that there are three types of reviewer comment: (1) where there is a problems - and a straightforward resolution; (2) where there seems to be a problem, but what it is and how to resolve it are unclear-you have not written clearly and your message has not been understood so, a significant rewrite is required; and (3) where you will likely be angry as the reviewer seems either not to have understood you or not to have read the paper properly-again a serious rewrite is needed, but be polite so that you don't add to the problem.

This book contains nearly everything you should know about scientific writing. Final sections deal with matters not covered elsewhere in the book, such as managing co-authorship and writing different types of articles. It ends with a chapter asking if scientific writing can be enjoyed, and can it include whimsy, humour and beauty? Read this book for the answers.

\section{CONFLICT OF INTEREST}

The author declares no conflict of interest.

Christopher Geoffrey Woods

The Clinical Medical School, University of Cambridge, Addenbrookes Hospital, Cambridge, UK E-mail:cw347@cam.ac.uk

\title{
Thorough discussion of cancer research-thoughts against the main stream
}

\author{
'Debating Cancer, The Paradox in Cancer Research', \\ Henry H Heng, Wayne State University, \\ School of Medicine, USA \\ ISBN: 9789814520843 \\ Published by: World Scientific: 2016 \\ Price: $£ 130.00 / £ 110.00$
}

European Journal of Human Genetics (2017) 25, 902-903; doi:10.1038/ejhg.2017.7

Cancer is, across the world, one among the five leading causes of death in humans. Thus, not only in USA but also elsewhere, cancer has been declared the 'biggest enemy of mankind', and during the past 30-50 years billions of private and governmental research money has been spent in the fight against cancer. While chromosomal aberrations and viral infections (oncogenes) were initially the research focus, for several decades the main attention has shifted to the 'gene mutation theory of cancer'. It is common sense that (a) there is a linear accumulation of mutations in a cell that stepwise evolves to a cancer cell; (b) mutations are different but specific for each cancer type; (c) leukemia behaves principally as solid tumors; and (d) once we accomplish a detailed study of each type of cancer, we will be able to find its Achilles heel and develop a drug against that type. A standard (and, notably, still almost only) example is that of chronic myelogenous leukemia (chronic phase), with $B C R-A B L$ gene fusion and Imatinib as a cure.

In his book Prof Heng acknowledges and summarizes all these historical developments of cancer research in a comprehensive and entertaining way. However, he also points out clearly that something went wrong in this research field at least during the last two decades. The book, surely mind-blowing for many readers, starts with the quotation of Dr Harold Varmus (former director of US National Cancer Institute), who wrote in his resignation letter concerning the current status of cancer research, perhaps including the 'gene mutation theory of cancer' and the yet widely unsuccessful ( $>5$ years) attempts to find common driver oncogenes by next-generation sequencing approaches: 'We've run out of money, it is time to start thinking'!

The book Debating Cancer, The Paradox in Cancer Research is an excellent example of how critical scientific thinking needs to be done, and the author invites the reader to and takes him on a journey of many unsolved questions and problems in this important field. In the first two chapters the present problem of the cancer research, being trapped by focusing only on the 'gene mutation theory of cancer', is outlined; Chapter 3 is about the existing alternative theories to explain cancer; and Chapter 4 reviews and discusses the somewhat disappointing practical results of the highly praised cancer genome sequencing project(s), and the inability to transfer the (often contradictory and/or 
confusing) results to the clinical settings. In Chapters 5 and 6 Prof Heng introduces the genome theory of cancer evolution and spreads ideas on the high impact of the yet underestimated tumor variability, and introduces chromosome instability (CIN), chromothripsis, genomic chaos, fuzzy genomes and the two-step evolution model of cancer. By differentiating gene-defined 'parts inheritance' and genome-defined 'system inheritance' or blueprint, these two chapters illustrate the ultimate importance of genome (karyotype) heterogeneity in cancer research, as the package of altered genomes, rather than individual cancer gene mutations, drive the macro-evolution of cancer. In case the reader is puzzled before coming to Chapter 5 as to what the book reaches out for, he can jump to Fig 5.4 and its legend. Chapter 7 acknowledges the fact that cancer might be interpreted as being similar in development to speciation and, finally, with all that in mind one is ready to proceed to an overall discussion of the problems of cancer research in Chapter 8 and the Epilogue.

Overall, Prof Heng has written an excellent summary and highlights impressively the importance of considering all sides of a problem, including such that do not fit into the mainstream theory, for example, single-cell aberrations in cancer and high rates of CIN. What lacks more or less completely is the discussion of cancer development and its similarities to embryogenesis; while it is mentioned that oncogenes are growth and developmental genes, the fact that chromothripsis and CIN can be found in the early stages of fetal development as in cancer is not covered here. However, by referring to Albert Einstein's statement that if he were 'given 20 days to solve a problem, he would spend 19 days defining it', Prof Heng focusses clearly on what he wants to achieve with this book: create discussions on how to continue cancer research best, and to find better theories than 'gene mutation theory' to make tumors accessible and curable in the end.

In conclusion, this book should be read by each one working in cancer research, diagnostics and treatment, including students of medicine, biology and bioinformatics.

\section{CONFLICT OF INTEREST}

The author declares no conflict of interest.

Thomas Liehr

Jena University Hospital, Friedrich Schiller University, Institute of Human Genetics, Jena, Germany E-mail:Thomas.Liehr@med.uni-jena.de 